\title{
Qualitative Behavior of Dissipative Wave Equations on Bounded Domains
}

\author{
JEFFREY RAUCH
}

\author{
Communicated by C. DAFERMos
}

\begin{abstract}
The qualitative behavior of solutions of the mixed problem $u_{t t}=\Delta u-a(x) u_{t}$ in $\mathbb{R} \times \Omega, u=0$ on $\mathbb{R} \times \partial \Omega$, is studied in the case when $a>0$ and $\Omega \subset \mathbb{R}^{n}$ is bounded. Roughly speaking, if $a \geqq a_{\min }>0$, then solutions decay at least as fast as $\exp t\left(\varepsilon-\frac{1}{2} a_{\min }\right)$, with the possible exception of a finite dimensional set of smooth solutions whose existence is associated with a phenomenon of overdamping. If $a_{\max }$ is sufficiently small, depending on $\Omega$, then no overdamping occurs.

Let $\Omega \subset \mathbb{R}^{n}$ be a bounded open set lying on one side of its smooth boundary $\partial \Omega$. We are interested in solutions of the dissipative wave equation
\end{abstract}

$$
u_{t t}=\Delta u-a(x) u_{t} \quad \text { on } \quad \mathbb{R} \times \Omega,
$$

where $\Delta$ is the Laplacian and where the coefficient of friction $a(x)$ is a smooth positive function on $\bar{\Omega}$. On the boundary the Dirichlet boundary condition

$$
u=0 \quad \text { on } \mathbb{R} \times \partial \Omega
$$

is imposed. The methods used generalize immediately to a wide class of equations and boundary conditions.

A feeling for the phenomena encountered can be obtained by considering the special case $a=$ constant, which can be solved by an eigenfunction expansion. Let $\Phi_{j}, j=1,2, \ldots$, be an orthonormal sequence of eigenfunctions of $\Delta$ with Dirichlet conditions. That is, $\Delta \Phi_{j}=\lambda_{j} \Phi_{j}$ in $\Omega, \Phi_{j}=0$ on $\partial \Omega$ and $0>\lambda_{1}>\lambda_{2} \geqq \lambda_{3} \ldots$. Then

$$
u=\sum u_{j}(t) \Phi_{j},
$$

where $u_{j}(t)$ satisfies the damped spring equation

$$
\ddot{u}_{j}+a \dot{u}_{j}-\lambda_{j} u_{j}=0
$$

with $u_{j}(0), \dot{u}_{j}(0)$ determined by the Cauchy data of $u$. The general solution of (4) is

$$
\begin{gathered}
\beta_{+} e^{r_{+} t}+\beta_{-} e^{r-t}, \quad \beta_{ \pm} \in \mathbb{C} \\
r_{ \pm}=\frac{-a \pm \sqrt{a^{2}+4 \lambda_{j}}}{2} .
\end{gathered}
$$

Partially supported by NSF grant NSF GP 34260 . 
Since $\lambda_{j} \rightarrow-\infty$ as $j \rightarrow \infty$ we see that for $j$ large $\operatorname{Re} r_{ \pm}=-a / 2$ and the corresponding terms in (3) decay like $e^{-t a / 2}$. The "slow modes" with $\operatorname{Re} r>-a / 2$ occur when the spring equation (4) is overdamped. In summary, solutions consist of a finite dimensional part decaying exponentially, but slower than $e^{-t a / 2}$, and a part decaying like $e^{-t a / 2}$. Our goal is to prove similar results when $a$ depends on $x$. To do this, an appropriate framework for discussing (1), (2) must be built.

The energy of a solution $u$ at time $t$ is defined as

$$
E(t, u)=\int_{\Omega}\left(\left|u_{t}(t, x)\right|^{2}+|\nabla u(t, x)|^{2}\right) d x .
$$

The basic energy identity for smooth solutions of (1), (2) is then

$$
\frac{d}{d t} E(t, u)=-2 \int_{\Omega} a(x)\left|u_{t}(t, x)\right|^{2} d x \leqq 0 .
$$

This relation would seem to indicate that if one increases $a$ then the energy should decay more rapidly. It was pointed out in [5, pp. 365-367] however that this is not quite correct, since for large $a$ the overdamped states actually decay slower. With the exception of this finite dimensional set of states, however, the idea is right.

We introduce the natural Hilbert space of states, $H=\stackrel{\circ}{H}_{1}(\Omega) \times L_{2}(\Omega)$ with norm

$$
\|(\phi, \psi)\|_{H}^{2}=\int_{\Omega}\left(|\nabla \phi|^{2}+|\psi|^{2}\right) d x
$$

and define the evolution operator $S(t): H \rightarrow H$ by

$$
S(t)(\phi, \psi)=\left(u(t), u_{t}(t)\right),
$$

where $u$ is the solution of the mixed problem (1), (2) with initial conditions

$$
u(0)=\phi, \quad u_{t}(0)=\psi .
$$

(If one wants to avoid weak solutions one may consider functions $(\phi, \psi) \in C_{0}^{\infty}(\Omega)^{2}$, in which case the solution of $(1),(2),(7)$ is smooth and $S(t)$ has a unique continuous linear extension to all of $H$.) The family $S(t),-\infty<t<\infty$, is a $C_{0}$ one-parameter group of linear transformations on $H$ with $\|S(t)\| \leqq 1$ for $t \geqq 0$. The generator of this group is

$$
\begin{gathered}
G=\left(\begin{array}{ll}
0 & 1 \\
\Delta & 0
\end{array}\right)+\left(\begin{array}{cc}
0 & 0 \\
0 & -a
\end{array}\right), \\
D(G)=\left(H_{2}(\Omega) \cap \stackrel{\circ}{H}_{1}(\Omega)\right) \times \stackrel{\circ}{H}_{1}(\Omega) .
\end{gathered}
$$

Notice that $\left(\begin{array}{ll}0 & 1 \\ \Delta & 1\end{array}\right)$, with the same domain, is skewadjoint (see $[4, \S \mathrm{V} .1]$ ) and that $\left(\begin{array}{cc}0 & 0 \\ 0 & -a\end{array}\right)$ is a bounded dissipative operator on $H$ so that $G$ is maximal dissipative.

We are interested in $S(t)$ for $t$ large. If $\Omega$ were a Riemannian manifold without boundary and $\Delta$ the Laplace-Beltrami operator, then the results of [7], see especially $\S 4$, give a precise formula for $\|S(t)\|_{\mathscr{L} / \mathscr{X}}$ where $\mathscr{L}$ is the algebra of 
bounded operators on $H$ and $\mathscr{K}$ the ideal of compact operators. In particular, $\|S(t)\|_{\mathscr{L} / \mathscr{K}}$ depends monotonically on $a$, in the sense that increasing a decreases the norm of $S(t)$. We have also the simple estimates

$$
e^{-t a_{\max } / 2} \leqq\|S(t)\|_{\mathscr{L} / \mathcal{K}} \leqq e^{-t a_{\min } / 2},
$$

where

$$
a_{\max }=\max _{\Omega} a, \quad a_{\min }=\min _{\Omega} a .
$$

The proof in [7] relies on a detailed construction using geometrical optics, not available in case $\Omega$ has a boundary because of the existence of glancing rays. We will give a direct and elementary proof of inequalities corresponding to (8) in the case of regions with boundary. The method is that of energy integrals with an indefinite energy form.

Definition. Let $\pi_{1}: H \rightarrow \stackrel{\circ}{H}_{1}(\Omega)$ be defined by $\pi_{1}((\phi, \psi))=\phi$. If $h \in H$ then $u=$ $\pi_{1}(S(t) h)$ is called a finite energy solution of (1), (2). Notice that

$$
u \in C\left(\mathbb{R} \mid \stackrel{\circ}{H}_{1}(\Omega)\right) \cap C^{1}\left(\mathbb{R} \mid L_{2}(\Omega)\right) .
$$

The next result contains our basic estimate.

Theorem 1. If $u$ is a solution of (1), (2) with finite energy, then

$$
E(t, u) \leqq c_{1}^{2} e^{-a_{\min } t} E(0, u)+c_{2}\|u(t)\|_{L_{2}}^{2},
$$

where

$$
\begin{aligned}
& c_{1}^{2}=\max \left(4, a_{\min }^{2} / 2 \lambda\right), \\
& c_{2}=a_{\max } a_{\min }-\lambda
\end{aligned}
$$

and $\lambda$ is given by (11).

One consequence of $(10)$ is that for solutions which decay slower than $e^{-t a_{\min } / 2}$ the energy is dominated by $\left(c_{2}+\varepsilon\right)$ times the $L_{2}$ norm, for large time. Thus the slowly decaying modes must be fairly smooth.

Proof. First assume that $u \in C^{\infty}(\mathbb{R} \times \bar{\Omega})$. Let $\alpha=a_{\min } / 2$ and $v=e^{\alpha t} u$. Then

$$
\begin{aligned}
u_{t} & =e^{-\alpha t}\left(v_{t}-\alpha v\right) \\
u_{t t} & =e^{-\alpha t}\left(v_{t t}-2 \alpha v_{t}+\alpha^{2} v\right) \\
\Delta v & =e^{\alpha t} \Delta u=e^{\alpha t}\left(u_{t t}+a u_{t}\right) .
\end{aligned}
$$

Substituting the expressions for $u_{t}, u_{t}$ into the last equation yields

$$
v_{t t}-\Delta v+\left(\alpha^{2}-a \alpha\right) v=-\left(a-a_{\min }\right) v_{t}
$$

(notice that $a-a_{\min } \geqq 0$ and $\alpha^{2}-a \alpha \leqq-\alpha^{2}<0$ ). Multiply this equation by $\bar{v}_{t}$, integrate over $\Omega$, and form the real part. This shows that

$$
\frac{d}{d t} \mathscr{I}(t)=-2 \int_{\Omega}\left(a-a_{\min }\right)\left|v_{t}(t)\right|^{2} d x \leqq 0,
$$


where

$$
\mathscr{I}(t)=\int_{\Omega}\left(\left|v_{t}(t)\right|^{2}+|\nabla v(t)|^{2}+\left(\alpha^{2}-a \alpha\right)|v(t)|^{2}\right) d x .
$$

Notice that the decreasing functional $\mathscr{I}(t)$ is indefinite since $\alpha^{2}-a \alpha<0$. To make use of the decrease of $\mathscr{I}$, observe that

$$
\begin{aligned}
e^{2 \alpha t} E(t, u) & =\int_{\Omega}\left(\left|v_{t}-\alpha v\right|^{2}+|\nabla v|^{2}\right) d x \\
& \leqq \int_{\Omega}\left(2\left|v_{t}\right|^{2}+2 \alpha^{2}|v|^{2}+|\nabla v|^{2}\right) d x \\
& \leqq 2 \mathscr{I}(t)+2 a_{\max } \alpha\|v(t)\|_{L_{2}}^{2}-\|\nabla v(t)\|_{L_{2}}^{2},
\end{aligned}
$$

where we have used the relation $2 a_{\max } \alpha+2\left(\alpha^{2}-a \alpha\right) \geqq 2 \alpha^{2}$. Let $\lambda$ be the smallest eigenvalue of $-\Delta$ with Dirichlet boundary conditions, that is

$$
\lambda=\min _{\phi \in H_{1}(\Omega) \backslash\{0\}} \frac{\|\nabla \phi\|_{L_{2}}^{2}}{\|\phi\|_{L_{2}}^{2}} .
$$

Then the decrease of $\mathscr{I}$ and the inequality for $E$ yield

$$
E(t, u) \leqq 2 e^{-2 \alpha t} \mathscr{I}(0)+\left(a_{\max } a_{\min }-\lambda\right)\|u(t)\|_{L_{2}}^{2} .
$$

In addition,

$$
\begin{aligned}
\mathscr{I}(0) & =\int_{\Omega}\left(\left|u_{t}(0)+\alpha u(0)\right|^{2}+|\nabla u(0)|^{2}+\left(\alpha^{2}-a \alpha\right)|u(0)|^{2}\right) d x \\
& \leqq E(0, u)+\int_{\Omega}\left(\left|u_{t}(0)\right|^{2}+\left(3 \alpha^{2}-a \alpha\right)|u(0)|^{2}\right) d x \\
& \leqq \max \left(2, \frac{\alpha^{2}}{\lambda} E(0, u)\right),
\end{aligned}
$$

where we have used the estimate $3 \alpha^{2}-a \alpha \leqq \alpha^{2}$. Substituting the above estimate for $\mathscr{I}(0)$ into the inequality for $E(t, u)$ yields $(10)$.

If $u$ is a solution with finite energy, say $u=\pi_{1}(S(t) h)$, we choose $h_{n}=\left(\phi_{n}, \psi_{n}\right) \in$ $\left(C_{0}^{\infty}(\Omega)\right)^{2}$ with $\left(\phi_{n}, \psi_{n}\right) \rightarrow h$ in $H$, and let $u_{n}=\pi_{1} S(t) h_{n}$. Then $u_{n} \in C^{\infty}(\mathbb{R} \times \bar{\Omega})$ and $E\left(t, u_{n}\right) \rightarrow E(t, u)$ by virtue of the continuity of $S$. Applying inequality (10) to $u_{n}$ and passing to the limit $n \rightarrow \infty$ proves the theorem.

Theorem 1 has strong implications for the spectrum of $S(t)$. On $H$, let || be the continuous seminorm defined by

$$
|(\phi, \psi)|^{2}=\int_{\Omega}|\phi|^{2} d x .
$$

This seminorm is compact in the following sense.

Definition. If $Y$ is a Banach space and $p: Y \rightarrow \mathbb{R}$ is a continuous seminorm, then $p$ is compact if every bounded sequence $\left\{y_{n}\right\}$ in $Y$ has a subsequence $\left\{y_{n_{k}}\right\}$ such that $p\left(y_{n_{k}}-y_{n_{l}}\right) \rightarrow 0$ as $k, l \rightarrow \infty$.

Notice that if $\left\{\left(\phi_{n}, \psi_{n}\right)\right\}$ is bounded in $H$, then $\left\{\phi_{n}\right\}$ is bounded in $\stackrel{\circ}{H}_{1}(\Omega)$. Hence by Rellich's theorem there is a subsequence $\left\{\phi_{n_{k}}\right\}$ convergent in $L_{2}(\Omega)$. 
This proves the compactness of $\mid 1$. As a second observation note that if $A: Y \rightarrow Y$ is a continuous linear map and $p: Y \rightarrow \mathbb{R}$ is a compact seminorm, then $p \circ A$ is a compact seminorm. For any $t>0$, let $q: H \rightarrow \mathbb{R}$ be the seminorm defined by $q(h)=|S(t) h|$. The above remarks show that $q$ is compact. The basic estimate (10) shows that

$$
\|S(t) h\|_{H} \leqq c_{1} e^{-t a_{\min } / 2}\|h\|_{H}+\sqrt{c_{2}} q(h)
$$

Though this is not as strong as the estimate $\|S(t)\| \leqq$ const $e^{-t a_{\min } / 2}$, we will show that it is also not much weaker (see Theorem 4).

First we study the spectrum $\sigma(S(t))$ of $S(t)$. Recall that an isolated point $\lambda$ of $\sigma(S)$ is an eigenvalue of finite multiplicity if $[z-S]^{-1}$ has a pole at $z=\lambda$. The (geometric) multiplicity of $\lambda$ as an eigenvalue is the rank of the projection

$$
(2 \pi i)^{-1} \oint_{\Gamma}(z-S)^{-1} d z
$$

where $\Gamma$ is a circle enclosing $\lambda$ but no other part of $\sigma(S)$.

Theorem 2. Let $D$ be the disc $\left\{z|| z \mid \leqq c_{1} e^{-t a_{\min } / 2}\right\}$. Then $\sigma(S(t)) \cap \mathbb{C} \backslash D$ is a discrete subset of $\mathbb{C} \backslash D$ consisting only of eigenvalues of finite multiplicity.

Theorem 2 follows by applying the next lemma, which is a standard Fredholm type result (proof omitted).

Lemma. Suppose $H$ is a Hilbert space, $S: H \rightarrow H$ is a bounded linear transformation, and $q$ a compact seminorm on $H$. If

$$
\|S h\|_{H} \leqq C\|h\|_{H}+C^{\prime} q(h), \quad h \in H,
$$

then $\sigma(S) \cap\{|z|>C\}$ consists of isolated eigenvalues with finite multiplicity.

If $G$ is the generator of the group $S(t)$, then $\sigma(G) \subset\{\operatorname{Re} z \leqq 0\}$. The following more detailed result also holds.

Theorem 3. We have $\sigma(G) \subset\{\operatorname{Re} z<0\}$. Moreover for any $\varepsilon>0$ the set $\sigma(G) \cap$ $\left\{\operatorname{Re} z \geqq \varepsilon+a_{\min } / 2\right\}$ consists of a finite number of isolated eigenvalues of finite multiplicity.

Proof. The second assertion follows from Theorem 2 and the basic spectral theory of semigroups, for example, Theorems 16.7.1 and 16.7.2 of [3]. It remains to show that there are no purely imaginary eigenvalues. If $G h=i \mu h$ with $\mu \in \mathbb{R}$, and $h=(\phi, \psi) \in H$, then

$$
0=\operatorname{Re}(G h, h)=-2 \int_{\Omega} a|\psi|^{2} d x .
$$

Since $a>0$ we see that $\psi=0$. The equation $G h=i \mu h$ implies $\psi=i \mu \phi$. Hence if $\mu \neq 0$ it follows that $h=0$. If $\mu=0$ we have $G h=0$, so $\Delta \phi=a \psi$. Now $\psi=0$, whence $\phi \in \stackrel{\circ}{H}_{1}(\Omega)$ and $\Delta \phi=0$. Thus $\phi=0$ and again $h=0$. This completes the proof.

Fix $\varepsilon>0$ and let $\Gamma$ be a smooth simple closed curve enclosing the eigenvalues of $G$ with real parts greater than or equal to $\varepsilon-a_{\min } / 2$, but enclosing no other 
part of $\sigma(G)$. Let $P$ be the corresponding spectral projection, that is,

$$
P=\frac{1}{2 \pi i} \oint_{\Gamma}(z-G)^{-1} d z .
$$

By Theorem 3, $P$ has finite rank. The general spectral theory of closed operators shows that the decomposition $H=P H \oplus(I-P) H$ reduces $G$ and $S$ in the following sense:

(i) $S(t)$ maps $P H$ and $(I-P) H$ into themselves.

(ii) $P H \subset D(G)$, and the generator of the group $\left.S(t)\right|_{P H}$ is $\left.G\right|_{P H}$. The generator of $\left.S(t)\right|_{(I-P) H}$ is $\left.G\right|_{D(G) \cap(I-P) H}$.

(iii) $\sigma\left(\left.S(t)\right|_{P H}\right) \subset\left\{|z| \geqq e^{t\left(\varepsilon-a_{\min } / 2\right)}\right\}$

and

$$
\sigma\left(\left.S(t)\right|_{(I-P) H}\right) \subset\left\{|z| \leqq e^{t\left(\varepsilon^{\prime}-a_{\min } / 2\right)}\right\} \text { for some } \varepsilon^{\prime} \in[0, \varepsilon) .
$$

From this we derive the following basic decay theorems.

Theorem 4. For $\varepsilon>0$, let $P$ be the spectral projection of $G$ corresponding to the eigenvalues of $G$ with real part $\geqq-a_{\min } / 2+\varepsilon$. Then $H=P H \oplus(I-P) H, P H$ is finite dimensional, both $P H$ and $(I-P) H$ are invariant under $S(t)$, and there is an $\varepsilon^{\prime}<\varepsilon$ such that

$$
\left\|\left.S(t)\right|_{(I-P) H}\right\| \leqq C e^{t\left(\varepsilon^{\prime}-a_{\min } / 2\right)}, \quad t \geqq 0 .
$$

Proof. All that needs to be proved is the decay estimate. Let $\tilde{S}=\left.S(1)\right|_{(I-P) H}$. Then by (iii), $\sigma(\tilde{S}) \subset\{|z| \leqq \rho\}$ for some $\rho<e^{-a_{\min } / 2+\varepsilon}$. The spectral radius formula now implies

$$
\rho=\lim _{n \rightarrow \infty}\left\|\tilde{S}^{n}\right\|^{1 / n}=\lim \left\|\left.S(n)\right|_{(I-P) H}\right\|^{1 / n}
$$

It follows that

$$
\left\|\left.S(n)\right|_{(I-P) H}\right\| \leqq \text { const. } e^{\left(\varepsilon^{\prime}-a_{\min } / 2\right) n}
$$

for $n=0,1,2, \ldots$. Since $S$ is a contraction semigroup for $t \geqq 0$, we have

$$
\left\|\left.S(t)\right|_{(I-P) H}\right\| \leqq\left\|\left.S([t])\right|_{(I-P) H}\right\| \leqq \text { const. } e^{\left(\varepsilon^{\prime}-a_{\min } / 2\right)[t]}
$$

where [ ] is the greatest integer function. This inequality implies the desired estimate.

The above theorem renders precise the heuristic description of decay described in the introduction.

Corollary 5. If $r=\sup \{\operatorname{Re} z \mid z \in \sigma(G)\}$ and $r>-a_{\min } / 2$, then there is an integer $m$ and a real number $c$ such that for all $t \geqq 0$ we have

$$
\|S(t)\| \leqq c\left(1+t^{m}\right) e^{r t} .
$$

Proof. Choose $\varepsilon>0$ so that $\varepsilon-a_{\min } / 2<r$, and then write $H=P H \oplus(I-P) H$ as in Theorem 4. The estimate (14) suffices for the consideration of $\left.S\right|_{(I-P) H}$. How- 
ever, $P H$ is finite dimensional and $\sigma\left(\left.G\right|_{P H}\right) \subset\{\operatorname{Re} z \leqq r\}$, so for $t \geqq 0$

$$
\left\|\left.S(t)\right|_{P H}\right\| \leqq \text { const. }\left(1+t^{m}\right) e^{r t}
$$

with $m+1 \leqq \operatorname{dim} P H$.

Remark. It is a simple matter to derive decay estimates for the higher derivatives of $u$. For example, if $h \in D(G)$ and $u=\pi_{1}(S(t) h)$, then $u_{t}=\pi_{1}(S(t) G h)$ is also a finite energy solution of $(1),(2)$. Hence $E\left(t, u_{t}\right)$ decays exponentially, and in particular $\left\|u_{t}\right\|_{L_{2}}$ and $\left\|u_{t t}\right\|_{L_{2}}$ decay like $e^{-(r-\varepsilon) t}$. From equation (1) it follows that $\|\Delta u\|_{L_{2}}$ decays at the same rate. Thus $\|u\|_{H_{2}(\Omega)}=O\left(e^{-(r-\varepsilon) t}\right)$, since $\Delta: H_{2}(\Omega) \cap \stackrel{\circ}{H}_{1}(\Omega) \rightarrow$ $L_{2}(\Omega)$ is an isomorphism. In the same way, if $h \in D\left(G^{k}\right)$ then $\left\|D_{t, x}^{\beta} u\right\|_{L_{2}}=O\left(e^{-(r-\varepsilon) t}\right)$ for $|\beta| \leqq k+1$.

The occurence of eigenvalues of $G$ with real part greater than $-a_{\min } / 2$ is connected with overdamping. If $a_{\max }$ is sufficiently small, one might expect that these eigenvalues do not occur and that $\|S(t)\|=O\left(e^{t\left(\varepsilon-a_{\min } / 2\right)}\right)$ as $t \rightarrow \infty$ for any $\varepsilon>0$.

Theorem 6. Let $\lambda$ be the smallest eigenvalue of $-\Delta$ with Dirichlet boundary conditions. If

$$
a_{\min }\left(2 a_{\max }-a_{\min }\right)<4 \lambda
$$

then $\sigma(G) \subset\left\{\operatorname{Re} z \leqq-a_{\min } / 2\right\}$.

Remark. If $a$ is constant the above inequality becomes $4 \lambda>a^{2}$. Since $\lambda=-\lambda_{1}$ in the notation of (6), this is precisely the condition on $a$ needed to rule out overdamping.

Proof. Suppose there is a value $\mu$ with $\operatorname{Re} \mu>-a_{\min } / 2$ and an $h \in H \backslash\{0\}$ such that $G h=i \mu h$. If $h=(\phi, \psi)$ then $u=e^{\mu t} \phi$ satisfies (1), (2). We now apply the method of proof used in Theorem 1. Let $\alpha=a_{\min } / 2$ and $v=e^{\alpha t} u$. Then

$$
\begin{aligned}
e^{2 \alpha t}\|\nabla u\|_{L_{2}}^{2} & \leqq E(t, v) \\
& \leqq \mathscr{I}(t)+\left(\alpha a_{\max }-\alpha^{2}\right)\|v(t)\|_{L_{2}}^{2} \\
& \leqq \mathscr{I}(0)+\left(\alpha a_{\max }-\alpha^{2}\right) e^{2 \alpha t}\|u(t)\|_{L_{2}}^{2} .
\end{aligned}
$$

Since $h \neq 0$ and $G h=i \mu h$, it follows that $\phi \neq 0$. Hence $\|u(t)\|_{L_{2}} \neq 0$. Then as $t \rightarrow \infty$

$$
\frac{\|\nabla \phi\|_{L_{2}}^{2}}{\|\phi\|_{L_{2}}^{2}}=\frac{\|\nabla u\|_{L_{2}}^{2}}{\|u\|^{2}}=\alpha a_{\max }-\alpha^{2}+o(1) .
$$

Letting $t \rightarrow \infty$ we have

$$
\lambda \leqq \frac{\|\nabla \phi\|_{L_{2}}^{2}}{\|\phi\|_{L_{2}}^{2}} \leqq \alpha a_{\max }-\alpha^{2},
$$

that is, $4 \lambda \leqq a_{\min }\left(2 a_{\max }-a_{\min }\right)$.

Remark. If one applies estimate (10) to the solution $u$, this gives the weaker estimate $2 \lambda \leqq a_{\max } a_{\min }$. In particular, when $a$ is constant this does not give a sharp result. The reason is that in (10) the need to estimate $\left\|u_{t}\right\|_{L_{2}}^{2}$ introduces some leeway into the estimate for $\nabla u$. 
Based on Theorem 6 and the case $a=$ constant, we make the following conjecture:

If $\lambda_{N}$ is the $N$ th largest (counting multiplicity) eigenvalue of $-\Delta$ with Dirichlet boundary conditions, and if $a_{\max }^{2}<4 \lambda_{N}$, then there are at most $N$ eigenvalues (counting multiplicity) of $G$ with real part larger than $-a_{\min } / 2$.

We have been concerned with upper bounds for $S(t)$, and the theme has been that "essentially" $\|S(t)\| \leqq$ const. $e^{-t a_{\min } / 2}$. There is a corresponding lower bound which, somewhat surprisingly, is true without qualification.

Theorem 7. If $u$ is a finite energy solution of (1), (2), then there is a positive constant $c$ such that

$$
E(t, u) \geqq c e^{-a_{\max } t} E(0, u), \quad t \geqq 0 .
$$

Proof. Let $\alpha=a_{\max } / 2$ and $v=e^{\alpha t} u$. Then for $v$ we have the differential equation

$$
v_{t t}-\Delta v+\left(\alpha^{2}+a \alpha\right) v=\left(a_{\max }-a\right) v_{t} .
$$

Since $a_{\max }-a \geqq 0$ the functional

$$
J(t)=\int_{\Omega}\left(\left|v_{t}(t)\right|^{2}+|\nabla v(t)|^{2}+\left(\alpha^{2}+a \alpha\right)|v(t)|^{2}\right) d x
$$

is increasing in $t$. Notice also that $\alpha^{2}+a \alpha \geqq 0$, so the norms $J^{1 / 2}$ and $E(t, v)^{1 / 2}$ are equivalent. Thus, for positive constants independent of $t, u, v$, we have

$$
\begin{aligned}
e^{-a_{\max } t} E(t, u) & \geqq \text { const. } E(t, v) \\
& \geqq \text { const. } J(t) \geqq \text { const. } J(0) \\
& \geqq \text { const. } E(0, v) \geqq \text { const. } E(0, u) .
\end{aligned}
$$

In terms of $S$ this theorem asserts that

$$
\|S(t) h\|_{H} \geqq c e^{-t a_{\max } / 2}\|h\|_{H}
$$

for all $t \geqq 0$ and $h \in H$. Since $S(-t)=S(t)^{-1}$ this shows that $\|S(-t)\| \leqq c e^{t a_{\max } / 2}$ for all $t \geqq 0$.

Corollary 8. $\sigma(G) \subset\left\{z \mid \operatorname{Re} z \geqq-a_{\text {max }} / 2\right\}$.

Proof. For $\operatorname{Re} z<-a_{\max } / 2$ we have

$$
(G-z I)^{-1}=\int_{-\infty}^{0} e^{-z t} S(t) d t,
$$

an absolutely convergent integral central in the theory of semigroups (see [3]).

Note. This work was partially supported by the National Science Foundation under Grant No. GP 34260 .

\section{References}

1. Dafermos, C., Contraction semigroups and trend to equalibrium in continuum mechanics, to appear.

2. IWASAKI, N., Local decay of solutions for symmetric hyperbolic systems with dissipative and coercive boundary conditions in exterior domains, Publ. RIMS Kyoto U. 5, 193-218 (1969). 
3. Hille, E. and R. Phillips, Functional Analysis and Semigroups. AMS Colloquim Publications, vol. 31 (1957).

4. LAX, P. and R. Phillips, Scattering Theory. New York: Academic Press 1967.

5. RAUCH, J., Five problems: an introduction to the qualitative theory of partial differential equations, in Partial Differential Equations and Related Topics. Springer Lecture Notes in Mathematics 446, 355-369 (1975).

6. RAUCH, J. and M.TAYLOR, Exponential decay of solutions to symmetric hyperbolic systems on bounded domains. Indiana Math. J. 24, 79-86 (1974).

7. RAUCH, J. and M.TAYLOR, Decay of solutions to non-dissipative hyperbolic systems on compact manifolds. Comm. Pure Appl. Math. 28, 501-503 (1975).

\section{Department of Mathematics University of Michigan Ann Arbor, Michigan}

(Received October 24, 1975) 